\title{
Section introduction \\ Introduction to sessions on 'Optimizing the implementation of future treatments - time to abandon traditional methods'
}

\author{
Mark Verrill
}

Northern Centre for Cancer Treatment, Newcastle General Hospital, Westgate Road, Newcastle upon Tyne, NE4 6BE, UK

Corresponding author: Mark Verrill, Mark.Verrill@nuth.nhs.uk

Published: 18 December 2008

This article is online at http://breast-cancer-research.com/content/10/S4/S21 (c) 2008 BioMed Central Ltd

This session highlighted current issues in clinical trials and, in particular, the importance of designing trials that are appropriate to the newer biological agents targeting specific cellular processes in tumours. Per Lønning described the different phases of clinical trials from I to III. He highlighted the importance of conducting extensive pharmacokinetic analyses during trials and of intensive characterization of drugs and their effects from the earliest phases of development. There are many ways in which this can be achieved, including pharmacodynamic assessments of drug effect and more complex gene chip assays, which can be used to predict who will be responders and nonresponders to particular treatments. Kathy Pritchard grappled with challenges faced by current trials, including the overbearing burden of regulation, the high costs of conducting trials in the host institution and the 'publish or die' mentality of academia. It is increasingly difficult to raise funds for trials, and 'sexy' research topics risk being prioritized ahead of the clinically relevant or those using older technologies. Clinical trial designs must be relevant to the whole population with the disease, not just the trial population, and the clinical indications for new drugs should not be limited by slavish application of restrictive trial entry criteria when the results are applied to the population.

lan Smith and Mitch Dowsett described a series of trials conducted at the Royal Marsden Hospital of preoperative exposure to drugs. Neoadjuvant treatment allows assessment of drug effects with the primary tumour in situ, but the key question is whether the observed treatment effect in this setting translated into improved outcomes in the longer term. Clinical response appears to correlate poorly with long-term outcome, but pathological complete response can be used to identify a group with better prognosis. The problem is that there are few pathological responses in the majority of neoadjuvant studies. Change in Ki67 staining after short-term exposure to drug does, however, appear to correlate better with longer-term outcome than does clinical response, and this might be a useful surrogate marker. Professor Dowsett
Breast Cancer Research 2008, 10(Suppl 4):S21 (doi:10.1186/bcr2181)

explained how Ki67 could better identify biological changes than tumour shrinkage because it takes into account the rate of proliferation at baseline. Thus, a rapidly growing tumour that appeared to respond only poorly clinically might actually have been dramatically 'slowed down', based on assessment of Ki67. Short-term exposure to drug before a traditional primary surgical approach may give useful biological information, and this approach is being tested in the POETIC (Pre-Operative Endocrine Therapy Individualised Care) study. Carlos Arteaga described how understanding of the mammalian target of rapamycin (mTOR) pathway in brain tumours could lead to prediction of outcome and the importance of translational, bench-to-bedside research in elucidating the interactions of drugs with complex growth signalling pathways.

In conclusion, in this session the importance of careful trial design of relevance to patients was emphasized. There should be extensive study of all aspects of drug action and metabolism in nonrandomized studies, so that when we embark on phase III trials including thousands of patients we know as much as possible about the effects of the drug. We are, as yet, some way off eliminating the need for these massive trials, because surrogate markers of outcome do not allow us to identify with sufficient precision those who will or will not benefit from an intervention. We must also be careful that the few per cent of cancer patients who do enter trials are relevant to the many who do not. One of the greatest challenges we face is applying the findings from the small subset of trial participants to all of the individuals in the population of breast cancer patients.

\section{Competing interests}

The author declares that they have no competing interests.

\section{Acknowledgement}

This article has been published as part of Breast Cancer Research Volume 10 Supplement 4, 2008: Controversies in Breast Cancer 2008. The full contents of the supplement are available online at http://breast-cancer-research.com/supplements/10/S4 